\title{
Fractional Revival of Rydberg Wave Packets in Twice-Kicked One-Dimensional Atoms
}

\author{
S. Chatterjee \\ Department of Physics, Bidhannagar College, EB-2, Sector-1, Salt Lake, Kolkata-700064, India
}

A. SAHA AND B. TALUKDAR*

Department of Physics, Visva-Bharati University, Santiniketan 731235, India

(Received January 28, 2011; in final form April 22, 2011)

\begin{abstract}
We study revival and fractional revival phenomena of wave packets in a one-dimensional Rydberg atom irradiated by two time-delayed half-cycle pulses using an autocorrelation function characterized by electronic transition probabilities as weighting factors rather than modeling them by a Gaussian or Lorentzian distribution. If the momentum $\left(q_{2}\right)$ delivered to the atom by the second kick is much smaller than that $\left(q_{1}\right)$ imparted by the first one, the times of revival and fractional revival coincide with those of the single kicked atom. For $q_{2} \geq \frac{q_{1}}{4}$ appearance of revival and fractional revival depends on both the values of $q_{2}$ and time delay $t_{1}$ between the pulses but more sensitively on $t_{1}$. The number of fractional revivals tends to become numerous as the value of $t_{1}$ increases.
\end{abstract}

PACS: 32.80.Rm, 03.65.Wj

\section{Introduction}

The Rydberg wave packets in atoms were first created by photoexcitation using ultrashort pulses [1, 2]. But since the pioneering experiment of Jones et al. [3], unipolar electric field pulses of tetrahertz spectrum, often called half-cycle pulses (HCPs), have been found to provide a very convenient tool to produce such packets. The duration of an HCP is much shorter than the Kepler period of the Rydberg electron such that during interaction the electron effectively receives an impulsive kick from the former. The HCP can have sufficient bandwidth to excite the neighbouring states of the kicked electron resulting in the formation of a wave packet. The Rydberg wave packets can be conveniently used to probe the correspondence between classical and quantum mechanics because of the following.

(i) Initially, the motion of the wave packet is periodic with the same classical period $t_{\mathrm{cl}}$ as that of a charged particle in a Coulomb field.

(ii) However, this motion persists only for a few cycles after which quantum interference effects cause the wave packet first to collapse and then to undergo a sequence of revivals.

The revivals of the wave packets occur due to recombination of collapsed wave packets at a time $t_{\text {rev }}$ into a form close to the original shape which again oscillates with pe$\operatorname{riod} t_{\mathrm{cl}}$. In addition, we can also have superrevival at a time $t_{\mathrm{sr}} \gg t_{\mathrm{rev}}$. For various times earlier than $t_{\mathrm{rev}}$ the wave packet gathers into a series of subsidiary wave pack-

\footnotetext{
* corresponding author; e-mail: binoy123@bsnl.in
}

ets so as to exhibit fractional revivals [4]. These occur at times equal to rational fractions of the full revival time.

The object of the present work is to study the revival structure of wave packets as arising in a one-dimensional Rydberg atom due to the impact of two half-cycle pulses with a time delay between their action. The one-dimensional atomic model is an excellent approximation for the Rydberg atom in the extreme Stark states and can faithfully mimic many properties of three-dimensional atoms [5]. Moreover, in this case one can construct an exact analytical expression for the wave packet irrespective of whether it is formed by the impact of a single HCP or two time delayed HCPs $[6,7]$. The shape of a wave packet can be controlled using a train of HCPs [8]. Recently, we found that for twice-kicked atoms the shape depends sensitively on the time delay $t_{1}$ between the kicks as well as the relative magnitudes of the momenta $q_{1}$ and $q_{2}$ transferred to the atomic electron by the HCPs [9]. It, however, remains an interesting curiosity to critically examine how the revival structure of the Rydberg wave packet depends on these parameters. The present work is an effort in this direction. We shall pay special attention on fractional revivals.

In Sect. 2, we present an expression for the wave packet formed by the impact of two time delayed HCPs on a one-dimensional atom. Such an expression could be conveniently used to control the shape of the wave packet as well as to demonstrate its revival properties [9]. But it cannot be used to look at the formation of mini packets corresponding to fractional revivals. The occurrence of these subsidiary packets can be realized by taking recourse to the use of so-called autocorrelation function [4] defined as an overlap of the initial wave packet with the 
corresponding wave packet at a later time $t$. The autocorrelation function gives a striking illustration of the different types of revival structures including the fractional ones. Numerically, the absolute square of the autocorrelation function varies between 0 and 1 and its periodicities reveal the periodic behaviour of the wave packet. Keeping this in view, we make use our result for the wave packet to express the autocorrelation function in terms of the momenta transferred to the atomic electron by two half-cycle pulses as well as time delay between them. Studies in fractional revivals for single kicked atoms is a well studied problem [10]. But to our knowledge there do not exist similar studies for twice kicked atoms. We feel that it is highly interesting to investigate the influence of the second time delayed HCP on the revival structure produced by the impact of the first one. We also make some useful observation on the expression presented. We devote Sect. 3 to critically examine the effects of the second delayed HCP on the revival structure of the wave packet. We achieve this by plotting modulus square of the autocorrelation function versus time of evolution for different values of time delay and momentum transferred by the second pulse. Finally, we make some concluding remarks in Sect. 4.

\section{Wave packet and autocorrelation function}

When a sequence of two HCPs having time delay $t_{1}$ between them is applied to the initial state $|n\rangle$ of a one-dimensional Rydberg atom, we obtain a wave packet of the form [7]:

$$
\begin{aligned}
& \psi\left(x, t, t_{1}\right)=\sum_{n^{\prime}, n^{\prime \prime}} \mathrm{e}^{-\mathrm{i} E_{n^{\prime \prime}}\left(t-t_{1}\right)} T_{n^{\prime \prime} n^{\prime}}\left(q_{2}\right) \\
& \quad \times \mathrm{e}^{-\mathrm{i} E_{n^{\prime}} t_{1}} T_{n^{\prime} n}\left(q_{1}\right)\left|n^{\prime \prime}\right\rangle,
\end{aligned}
$$

where $q_{1}$ and $q_{2}$ are the momenta transferred to the initial and intermediate states of the electron by the consecutive pulses. An interesting feature of the expression in (1) is that in addition to the usual summation over the final states $\left|n^{\prime \prime}\right\rangle$ which are believed to form the wave packet, it also involves a sum over all intermediate states $\left|n^{\prime}\right\rangle$ between $|n\rangle$ and $\left|n^{\prime \prime}\right\rangle$. The generic form of the transition matrices $T_{n^{\prime} n}\left(q_{1}\right)$ and $T_{n^{\prime \prime} n^{\prime}}\left(q_{2}\right)$ is given by [11]:

$$
\begin{aligned}
& T_{n^{\prime} n}(q)=\left\langle n^{\prime}\left|\mathrm{e}^{\mathrm{i} q x}\right| n\right\rangle=\frac{-z(\lambda-2 / n)^{n}\left(\lambda-2 / n^{\prime}\right)^{n^{\prime}}}{\sqrt{n^{3} n^{\prime 3}} \lambda^{n+n^{\prime}}} \\
& \quad \times\left[\left(\frac{n-1}{\lambda-2 / n}+\frac{n^{\prime}-1}{\lambda-2 / n^{\prime}}-\frac{n+n^{\prime}}{\lambda}\right)\right. \\
& \quad \times{ }_{2} F_{1}\left(-n+1,-n^{\prime}+1 ; 2 ; z\right)-\frac{(n-1)\left(n^{\prime}-1\right)}{2} \\
& \quad \times\left(\frac{1}{\lambda-2 / n}+\frac{1}{\lambda-2 / n^{\prime}}\right) z \\
& \left.\quad \times{ }_{2} F_{1}\left(-n+2,-n^{\prime}+2 ; 3 ; z\right)\right],
\end{aligned}
$$

$$
\lambda=\left(\frac{1}{n}+\frac{1}{n^{\prime}}-\mathrm{i} q\right)
$$

and

$$
z=-\frac{4 n^{\prime} n}{\left(n-n^{\prime}\right)^{2}+q^{2} n^{2} n^{\prime 2}} .
$$

Here ${ }_{2} F_{1}($.$) represents a Gaussian hypergeometric func-$ tion. From Eq. (1) it is clear that the wave packet $\psi\left(x, t, t_{1}\right)$ is characterized by the transition matrix elements $T_{i j}(q)$, where $i$ and $j$ refer to principal quantum numbers of either the kicked states or states which are coherently excited. From $T_{i j}(q)=\left\langle i\left|\mathrm{e}^{\mathrm{i} q x}\right| j\right\rangle$ it is evident that $T_{i j}(q)$ should be one for $i=j=n$ (say) and $q=0$. But the transition matrix element (2) in this case becomes undefined because the argument $z$ of the Gaussian hypergeometric functions becomes infinity. This difficulty can, however, be removed by writing $T_{n^{\prime} n}(q)$ in an alternative form given by [9]:

$$
\begin{aligned}
& T_{n^{\prime} n}=\alpha_{n^{\prime} n} \sum_{r^{\prime}=0}^{n^{\prime}-1} \beta_{r^{\prime} n^{\prime}} f_{m^{\prime} n^{\prime} n}(q), \quad \alpha_{n^{\prime} n}=\frac{4}{\sqrt{n^{3} n^{\prime 3}}}, \\
& m^{\prime}=r^{\prime}+3 .
\end{aligned}
$$

Here

$$
\beta_{r^{\prime} n^{\prime}}=\left(-\frac{2}{n^{\prime}}\right)^{r^{\prime}} \frac{1}{\Gamma\left(r^{\prime}+2\right)}\left(\begin{array}{c}
n^{\prime}-1 \\
r^{\prime}
\end{array}\right)
$$

and

$$
f_{m^{\prime} n^{\prime} n}(q)=\frac{\Gamma\left(m^{\prime}\right)}{y(q)^{m^{\prime}}}{ }_{2} F_{1}\left(-n+1,3+r^{\prime} ; 2 ; \frac{2}{n} y(q)^{-1}\right)
$$

with

$$
y(q)=\lambda .
$$

From Eqs. (5)-(8), it is easy to verify that $T_{n^{\prime} n}(0)=1$ for $n^{\prime}=n$ and 0 for $n^{\prime} \neq n$. We shall present all results of this work by making use of (5) rather than (2). In close analogy with the result for the single kicked atoms [10], the autocorrelation function for the twice kicked atoms can be written as

$$
A\left(t, t_{1}\right)=\left\langle\psi\left(x, 0, t_{1}\right) \mid \psi\left(x, t, t_{1}\right)\right\rangle .
$$

Using (1) in (9) we get

$$
\begin{aligned}
& A\left(t, t_{1}\right)=\sum_{n^{\prime} n^{\prime \prime} n^{\prime \prime \prime}} \mathrm{e}^{-\mathrm{i} E_{n^{\prime \prime}} t} T_{n^{\prime \prime} n^{\prime \prime \prime}}^{*}\left(q_{2}\right) T_{n^{\prime \prime \prime} n}^{*}\left(q_{1}\right) \\
& \quad \times T_{n^{\prime \prime} n^{\prime}}\left(q_{2}\right) T_{n^{\prime} n}\left(q_{1}\right) \mathrm{e}^{\mathrm{i} t_{1}\left(E_{n^{\prime \prime \prime}}-E_{n^{\prime}}\right)} .
\end{aligned}
$$

From the expression in (10) it is apparent that the coherently excited final states which form the wave packet have been denoted by the set $\left\{\left|n^{\prime \prime}\right\rangle\right\}$. For a particular value of $n^{\prime \prime}$, the state $\left|n^{\prime \prime}\right\rangle$ can be reached via two different intermediate states $\left|n^{\prime}\right\rangle$ and $\left|n^{\prime \prime \prime}\right\rangle$ as resulting from the impact of the first HCP. The autocorrelation function in (10) will depend on the time delay only if $n^{\prime} \neq n^{\prime \prime \prime}$ else it becomes independent of $t_{1}$.

The $T$ matrices $T_{n^{\prime} n}$ and $T_{n^{\prime \prime \prime} n}$ are strongly centered around the kicked state $n$. As a result those states with $E_{n^{\prime}}$ and $E_{n^{\prime \prime \prime}}$ near the value $E_{n}$ enter appreciably into 
the sum in (10). This permits expansion of $E_{n^{\prime}}$ and $E_{n^{\prime \prime \prime}}$ in a Taylor series about the energy of the centrally excited state $|n\rangle$. We thus write

$$
E_{n^{\prime}}=E_{n}+\frac{m_{1}}{n^{3}}-\frac{3 m_{1}^{2}}{2 n^{4}}
$$

and

$$
E_{n^{\prime \prime \prime}}=E_{n}+\frac{m_{3}}{n^{3}}-\frac{3 m_{3}^{2}}{2 n^{4}}
$$

with

$$
E_{n}=-\frac{1}{2 n^{2}}, \quad m_{1}=n^{\prime}-n, \quad \text { and } \quad m_{3}=n^{\prime \prime \prime}-n .
$$

Equations (11) and (12) can be written in terms of derivatives, $E_{n}^{i}=\frac{\mathrm{d}^{i} E_{n}}{\mathrm{~d} n^{i}}, i=1,2,3, \ldots$, so as to introduce distinct time scales that depend on $n$. We write

$$
\begin{aligned}
& t_{\mathrm{cl}}=\frac{2 \pi}{\left|E_{n}^{\prime}\right|}, \quad t_{\mathrm{rev}}=\frac{2 \pi}{\frac{1}{2}\left|E_{n}^{\prime \prime}\right|} \quad \text { and } \quad t_{\mathrm{sr}}=\frac{2 \pi}{\frac{1}{6}\left|E_{n}^{\prime \prime \prime}\right|}, \\
& E_{n}^{\prime}=E_{n}^{1}, \quad E_{n}^{\prime \prime}=E_{n}^{2} \quad \text { etc. }
\end{aligned}
$$

Here $t_{\mathrm{cl}}$ is the classical Kepler period. The second time scale $t_{\text {rev }}$ is the revival time. The third time scale is the superrevival time. The results in (14) refer strictly to the time scales applicable to single kicked atom since $T_{n^{\prime} n}$ or $T_{n^{\prime \prime \prime} n}^{*}$ corresponds to the $T$ matrix or weight factor associated with the wave packet resulting from the impact of first HCP. In a twice kicked atom $t_{\mathrm{rev}}$ and $t_{\mathrm{sr}}$ will be affected by the time delay $t_{1}$ as well as by a contribution from the exponential in (10). For $n^{\prime \prime \prime}=n^{\prime}$, the autocorrelation function in (10) will become independent of $t_{1}$. Also we note that for $q_{2}=0, T_{n^{\prime \prime} n^{\prime \prime \prime}}^{*}$ and $T_{n^{\prime \prime} n^{\prime}}$ can be replaced by $\delta_{n^{\prime \prime} n^{\prime \prime \prime}}$ and $\delta_{n^{\prime \prime} n^{\prime}}$ such that the autocorrelation function of the twice kicked atom goes over to that for the single kicked atom. This observation is likely to provide a useful guide in presenting results for $A\left(t, t_{1}\right)$, which we shall use to investigate the effects of second $\mathrm{HCP}$ on the revival structure.

\section{Revival times of the wave packet}

In this section we shall make use of Eqs. (10)-(13) to study revival properties of a wave packet produced by the impact of two time delayed HCPs on a one-dimensional Rydberg atom. We begin by noting that for $q_{2}=0$, $A\left(t, t_{1}\right) \rightarrow A(t)$, the autocorrelation function of a single kicked atom. The expression for $A(t)$ is given by

$$
A(t)=\sum_{n^{\prime}} \mathrm{e}^{-\mathrm{i} E_{n^{\prime}} t}\left|T_{n^{\prime} n}\right|^{2} .
$$

In general, the transition probabilities to different $\left|n^{\prime}\right\rangle$ states depend on the parameter $s=q n^{2}$ which is of the order of unity in the weak field limit. In this case only a few neighboring states are populated and a typical revival phenomenon is observed. But to study fractional revivals by the use of autocorrelation functions one usually needs to work with $s=5$ and $n=50$ [10] so that $q_{1}=0.002$ a.u. Using this value of $q_{1}$ and $E_{n^{\prime}}$ of (11) we computed the numbers for the autocorrelation function
$A(\tau)$ (single kicked atom) from (15) at different values of scaled time $\tau=\frac{t}{t_{\mathrm{cl}}}, t_{\mathrm{cl}}=2 \pi n^{3}$.

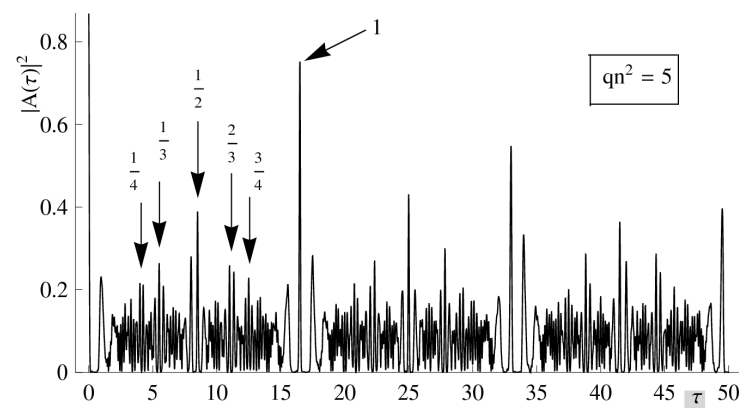

Fig. 1. Modulus square of the autocorrelation function, $|A(\tau)|^{2}$ showing fractional revivals as a function of scaled time $\tau$ for the momentum transfer $q=q_{1}=$ 0.002 a.u. in a single kicked atom.

Figure 1 gives $|A(\tau)|^{2}$ as a function of $\tau$. The highest peak of $|A(\tau)|^{2}$ occurs at $\tau=16.53\left(\approx \frac{n}{3}\right)$ and corresponds to the revival of the wave packet. The fractional revivals appear at times $\frac{\tau}{4}, \frac{\tau}{3}, \frac{\tau}{2}, \frac{2 \tau}{3}$ and $\frac{3 \tau}{4}$. The results of this figure are in agreement with those found by modelling the $T$ matrices by a Gaussian distribution with standard deviation $\sigma=3[10]$. We have verified that the use of Lorentzian distribution instead of the Gaussian also leads to similar results. However, we feel that it is always desirable to work in a purely quantum mechanical theory in which transition matrices occur in the expression for wave packet or autocorrelation function in a rather natural way. Keeping this in view, we made use of (10) to critically examine how the revival structures of Fig. 1 is affected by the action of the second time-delayed pulse.

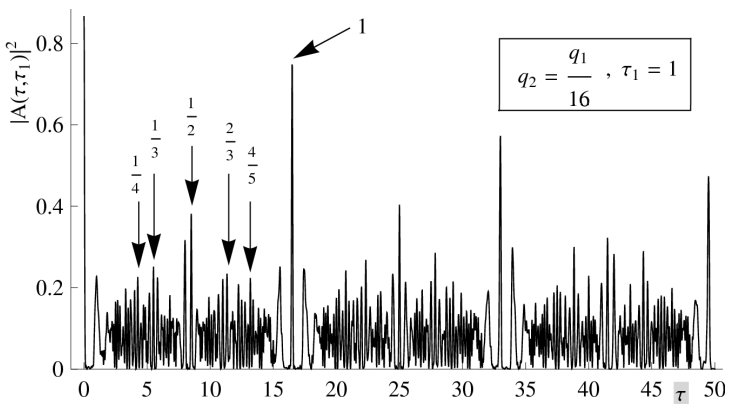

Fig. 2. Modulus square of the autocorrelation function, $\left|A\left(\tau, \tau_{1}\right)\right|^{2}$ showing fractional revivals as a function of scaled time $\tau$ in a twice kicked atom for $q_{1}=$ 0.002 a.u., $q_{2}=\frac{q_{1}}{16}$ and time delay $\tau_{1}=1$.

Figures 2-5 display the results for $\left|A\left(\tau, \tau_{1}\right)\right|^{2}$ computed from (10) as a function of $\tau$ for different values of $q_{2}$ and $\tau_{1}$. Let us note that, in addition to the continuous variable $\tau$, the autocorrelation function $A(\tau)$ depends on the discrete variable $q_{1}$. Similarly, for the twice kicked atom $A\left(\tau, \tau_{1}\right)$ depends on both discrete variables 


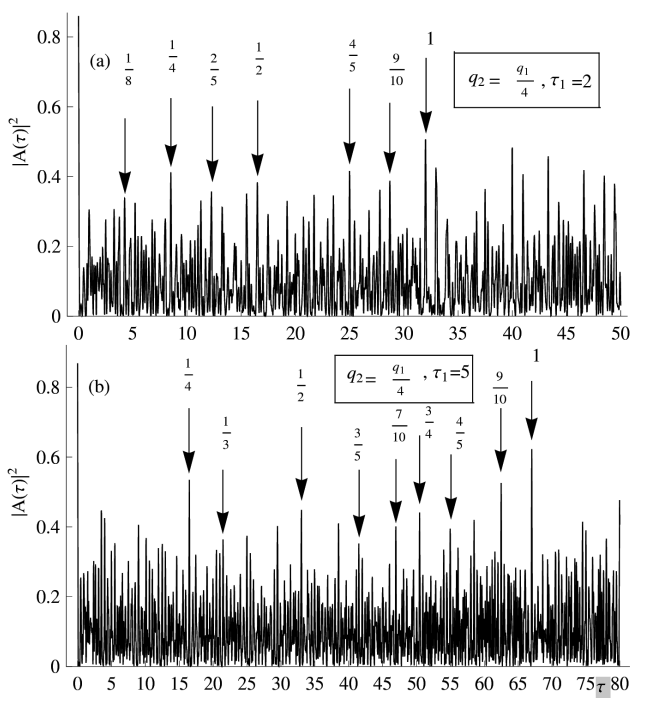

Fig. 3. The same as that in Fig. 2 but $q_{2}=\frac{q_{1}}{4}$, (a) $\tau_{1}=$ 2 and (b) $\tau_{1}=5$.

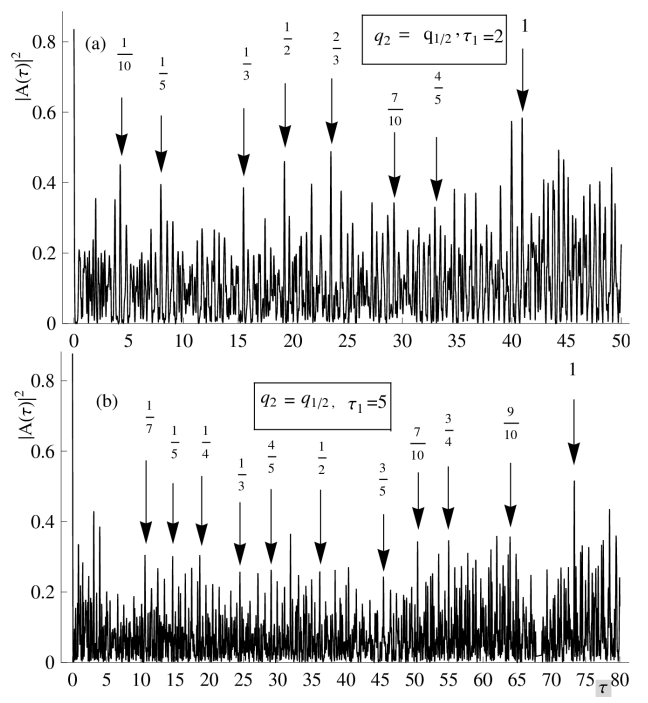

Fig. 4. The same as that in Fig. 2 but $q_{2}=\frac{q_{1}}{2}$, (a) $\tau_{1}=$ 2 and $(\mathrm{b}) \tau_{1}=5$.

$q_{1}$ and $q_{2}$. In Fig. 2, we present results for $\left|A\left(\tau, \tau_{1}\right)\right|^{2}$ for $q_{2}=\frac{q_{1}}{16}$ and $\tau_{1}=1$. For this value of $q_{2}$ and $\tau_{1}$, the revival structure of $\left|A\left(\tau, \tau_{1}\right)\right|^{2}$ is identical with that of $|A(\tau)|^{2}$ presented in Fig. 1. We have verified that for this small value of $q_{2}$ the revival structure of $\left|A\left(\tau, \tau_{1}\right)\right|^{2}$ remains unaltered for other higher values of $\tau_{1}$. The result is quite expected since an extremely weak second HCP may not have any effect on population distribution $\left(\left|T_{n^{\prime} n}\right|^{2}\right)$ such that $\left|A\left(\tau, \tau_{1}\right)\right|^{2} \approx|A(\tau)|^{2}$ for all values of $\tau_{1}$. But this may not be true when $q_{2}$ takes up larger values and tends towards $q_{1}$. To visualize how the results for $\left|A\left(\tau, \tau_{1}\right)\right|^{2}$ deviate from those for $|A(\tau)|^{2}$ as $q_{2} \rightarrow q_{1}$ we have plotted in Figs. 3-5 $\left|A\left(\tau, \tau_{1}\right)\right|^{2}$ as a function $\tau$ for $q_{2}=\frac{q_{1}}{4}, \frac{q_{1}}{2}, q_{1}$, respectively. In these figures the up-

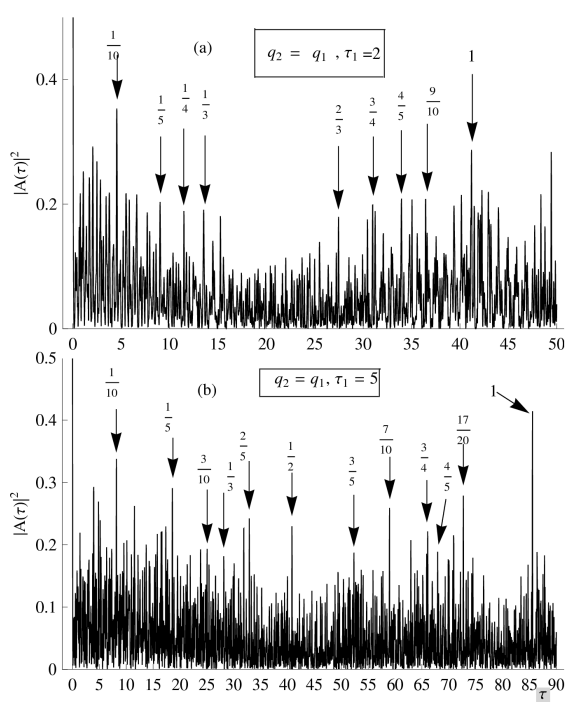

Fig. 5. The same as that in Fig. 2 but $q_{2}=q_{1}$, (a) $\tau_{1}=$ 2 and $(\mathrm{b}) \tau_{1}=5$.

per parts give the results for $\tau_{1}=2$ and the lower parts give those for $\tau_{1}=5$. Looking into the results in Fig. 3 we see that for $\tau_{1}=2$ the revival time of the wave packet is $\tau=32.03$. This number is approximately twice as that for the single kicked atom. For $\tau_{1}=5$ the revival of the wave packet occurs at a still large value of $\tau$, namely, $\tau=66.98$. Also we note that the number of fractional revivals for $\tau_{1}=2$ is six while that for $\tau_{1}=5$ is eight. Both numbers are bigger than the number of fractional revivals of the single kicked atom, the number for $\tau_{1}=5$ being larger than that for $\tau_{1}=2$. In general, the times of revival and numbers of fractional revival increase as the values of $q_{2}$ become large. For a given value of $q_{2}$ these numbers increase as $\tau_{1}$ becomes bigger. This can be verified by noting the times of revival and counting the number of fractional revivals as exhibited by the plots in Figs. 4 and 5.

From Figs. 2-5 it can be seen that as the value of $q_{2}$ is increased from $\frac{q_{1}}{16}$ to $q_{1}$ the heights of the peaks showing revivals and fractional revivals decrease roughly by $50 \%$. We verified that such diminutions increase as we go to still higher values of $q_{2}$ such that the revival structure might become physically unobservable. However, it is apparent from our study that for large $q_{2}$ values the impact of the time delayed second HCP causes the wave packet to reappear at much later time than that would be observed in the absence of it and simultaneously induces fractional revivals, the times of which are also quite different from those found in case of single kicked atoms or twice kicked atoms with rather low value of the momentum transferred by the second HCP.

To summarize the results displayed in Figs. 3-5 we present in Table the revival times of the wave packet in a twice kicked atom for different values of time delay $\tau_{1}$ and momentum $q_{2}$ transferred by the second kick to the electron excited by the impact of the first one. The 
TABLE

Variation of scaled revival time with $q_{2}$ and $\tau_{1}$.

\begin{tabular}{c|c|c|c|c|c}
\hline \hline$q_{2} \backslash \tau_{1}$ & 1 & 2 & 3 & 4 & 5 \\
\hline$\frac{q_{1}}{16}$ & 16.53 & 16.53 & 16.53 & 16.53 & 16.53 \\
$\frac{q_{1}}{8}$ & 16.53 & 16.53 & 16.53 & 16.53 & 16.53 \\
$\frac{q_{1}}{4}$ & 16.53 & 32.03 & 56.07 & 66.98 & 66.98 \\
$\frac{q_{1}}{2}$ & 17.48 & 41.01 & 57.49 & 71.09 & 73.31 \\
$q_{1}$ & 29.34 & 43.2 & 59.01 & 75.84 & 85.61
\end{tabular}

first two rows of the table show that for small values of $q_{2}$ the revival time is independent of both $\tau_{1}$ and $q_{2}$. This was why we presented plot of $\left|A\left(\tau, \tau_{1}\right)\right|^{2}$ only for $q_{2}=\frac{q_{1}}{16}$ (Fig. 2). As we go to the higher values of $q_{2}$, the revival times first increase with $\tau_{1}$ and then tend to take up a constant value. Looking closely into the numbers of this table we see that the revival times depend more sensitively on $\tau_{1}$ than on $q_{2}$ when $q_{2} \geq \frac{q_{1}}{4}$. For example, in the third row $\left(q_{2}=\frac{q_{1}}{4}\right)$ of the table we see that as $\tau_{1}$ goes from 1 to 5 the revival time changes by roughly $50 t_{\mathrm{cl}}$. The results in rows 4 to 5 also exhibit similar changes. On the other hand, for a fixed value of $\tau_{1}$ the change in revival time is relatively small as we move from lower to higher $q_{2}$ values. This is true for every column in Table.

\section{Conclusion}

We presented an expression for the autocorrelation function $A\left(t, t_{1}\right)$ for wave packets formed in a twice kicked one-dimensional Rydberg atom. Our expression (10) clearly shows that the final atomic states $\left|n^{\prime \prime}\right\rangle$ the superposition of which forms the wave packet can be reached via the intermediate states, namely, $\left|n^{\prime}\right\rangle$ and $\left|n^{\prime \prime \prime}\right\rangle$. For $n^{\prime}=n^{\prime \prime \prime}$, the autocorrelation function becomes independent of the time delay $t_{1}$ between the pulses since the last factor in (10) becomes unity. Thus the term in the summation of (10) having $n^{\prime}=n^{\prime \prime \prime}$ is physically undesirable. In view of this we omitted the contribution of this term in presenting all numerical results. We used the constructed expression for $A\left(t, t_{1}\right)$ to study the revival structure of the wave packet with special attention on the effect of the second HCP on the times of revival and fractional revivals. From the numerical results for $A\left(t, t_{1}\right)$ we could conclude that when the momentum delivered to the atom by the second HCP is not too small compared to that delivered by the first one, it is possible to manipulate the time delay $t_{1}$ between the pulses to observe wave packet revival at any desired time $t$. It is also possible to increase the number of fractional revivals by increasing the value of $t_{1}$.

\section{References}

[1] A. ten Wolde, L.D. Noordam, A. Lagendijk, H.B. van den Heuvell, Phys. Rev. Lett. 61, 2099 (1988).

[2] J.A. Yeazell, M. Mallalieu, J. Parker, C.R. Stroud Jr., Phys. Rev. A 40, 5040 (1989).

[3] R.R. Jones, D. You, P.H. Bucksbaum, Phys. Rev. Lett. 70, 1236 (1993).

[4] R. Bluhm, V.A. Kostelecky, J.A. Porter, Am. J. Phys. 64, 944 (1996).

[5] I. Bersons, R. Veilande, Phys. Rev. A 69, 043408 (2004).

[6] R. Parzynski, M. Sobczak, Phys. Rev. A 65, 045401 (2002).

[7] R. Parzynski, T. Kopyciuk, M. Sobczak, Phys. Lett. A 352, 505 (2006).

[8] M.W. Noel, C.R. Stroud Jr., Opt. Express 1, 176 (1997).

[9] A. Saha, S. Chatterjee, B. Talukdar, Phys. Scr. 81, 055302 (2010).

[10] R. Veilande, I. Bersons, J. Phys B, At. Mol. Opt. Phys. 40, 2111 (2007).

[11] L.D. Landau, E.M. Lifshitz, Quantum Mechanics, Pergamon, Oxford 1977. 